\title{
Application of a Two-Step Third-Derivative Block Method for Starting Numerov Method
}

\author{
Oluwaseun Adeyeye ${ }^{*}$, Zurni Omar \\ Department of Mathematics, Universiti Utara Malaysia, Kedah, Malaysia \\ Email address: \\ adeyeye_oluwaseun@ahsgs.uum.edu.my (O. Adeyeye), zurni@uum.edu.my (Z. Omar) \\ ${ }^{*}$ Corresponding author
}

\section{To cite this article:}

Oluwaseun Adeyeye, Zurni Omar. Application of a Two-Step Third-Derivative Block Method for Starting Numerov Method. International Journal of Theoretical and Applied Mathematics. Vol. 3, No. 1, 2017, pp. 30-35. doi: 10.11648/j.ijtam.20170301.15

Received: September 26, 2016; Accepted: December 10, 2016; Published: January 12, 2017

\begin{abstract}
Numerov method is one of the most widely used algorithms in physics and engineering for solving second order ordinary differential equations. The numerical solution of this method has been improved by different authors by using different starting formulas but in recent years, there has been a dearth in that trend which informed the introduction of a twostep third-derivative block method in this paper to start Numerov method with the aim of getting better results than previous approaches. The selection of the steplength as two is to have a uniform basis for comparison with other existing two-step starting formula in literature. Although, the accuracy of the two-step method adopted in this article was enhanced by the introduction of higher derivative. Hence, this paper presents a two-step third-derivative block method which displayed better accuracy when adopted for starting Numerov method as shown in the numerical results. Thus, the third-derivative block method, as a starting formula, is seen to be quite suitable for starting Numerov method when applied to physical models.
\end{abstract}

Keywords: Numerov, Two-Step, Third-Derivative, Block Method, Second Order, Initial Value Problems

\section{Introduction}

This paper considers the solution of the second order initial value problem of the form

$$
y^{\prime \prime}=f(x, y), y(a)=\alpha, y^{\prime}(a)=\beta
$$

where $\alpha$ and $\beta$ are constants.

Previously, the differential equations of the form (1) above have been solved by reduction to a system of two first order initial value problems. However, this approach involves both human and computational burden and hence the need to introduce direct numerical methods for the solution of (1) and the Numerov method is one of such direct methods.

The Numerov method as given below,

$$
y_{n+2}=2 y_{n+1}-y_{n}+\frac{h^{2}}{12}\left(f_{n}+10 f_{n+1}+10 f_{n+2}\right)
$$

is a very efficient two-step method for directly solving second-order differential equations of the form (1) and a suitable example is the Schrodinger equation as discussed by
$[7,15,17]$, and the Poisson equation as discussed in [11]. Also, some studies have shown that Numerov-type methods also being applied 2D Quasi-linear Elliptic Boundary Value Problems [10], 2D biharmonic and triharmonic equations [9] amongst many others. Hence, the importance of the Numerov method cannot be overemphasized and the presentation of a formula that will adequately start it to give better accuracy is an area of interest which is the aim of this paper.

The Numerov method has an order of accuracy of four with a corresponding error constant of $C_{6}=-\frac{1}{240}$. In the paper by [1], it was stated that this level of accuracy that the Numerov method possesses together with its application to (1) resulting in a tridiagonal set of algebraic equations has made the method popular. However, the question on how to adequately start the method and the quest to discover a better starting value dates back to the work of [6]. [6] observed that, for the application of the Numerov method in solving (1), there is a need for two previous values of the solution in order to calculate a new one. Hence, [6] adopted a starting formula of order three obtained using Taylor series approach.

[16] also developed a parallel algorithm with the aim of increasing the accuracy of the Numerov method. This 
algorithm was further extended by [1] who presented a formula for starting the Numerov method more accurately using an order four hybrid method developed based on multistep collocation approach to start the method. However, since the work of [1], there has been a dearth to the presentation of algorithms to adequately start the Numerov method which presents the area of interest in this paper.

The Numerov method, as widely known, is a two-step method and it is suitable to develop a two-step method to adequately start it with better accuracy. However, there is need to boost the two-step starting method with the introduction of higher derivative which gives rise to the twostep third-derivative method.

It is observed that very few contributions in literature exist on the application of higher derivative method; however, this method dates back to the early 40s as discussed by [3]. In his work, the author stated some particular cases of the introduction of second derivative in a first order linear multistep method which includes the method of order four considered by [12] and in connection with stiff equations by [2] amongst others. [3] developed a second derivative method to solve stiff first order ordinary differential equations and stated that the motivation for introducing the second derivative is to generate results with better accuracy when the method is applied to solve stiff first order initial value problems.

Similarly, in recent works, [5] developed a third-derivative method (TDM) to solve second order initial and boundary value problems and also, [14] developed a fourth derivative method (FDM) to solve third order boundary value problems. In their separate works, the introduction of higher derivative gave results with very good accuracy when compared with exact solution of the differential equation considered. Hence, the motivation to adopt a two-step higher derivative method for starting the Numerov method with expected accuracy.

This paper presents a third-derivative block method which is also a two-step method as the Numerov method for starting the Numerov method and obtaining better level of accuracy than previously proposed starting methods. The outline for this article is as follows: the derivation of the third-derivative block method is shown in Section 2 and its numerical results are shown in Section 3, the discussion of results is given in Section 4 and the article is concluded in Section 5.

\section{Derivation of the Third-Derivative Block Method}

The discrete scheme for the third-derivative block method is derived from the linear multistep method form given below

$$
y_{n+2}=\sum_{j=0}^{1} \alpha_{j} y_{n+j}+\sum_{j=0}^{2} \beta_{j} f_{n+j}+\sum_{j=0}^{2} \lambda_{j} g_{n+j}
$$

where

$$
f_{n+j}=f\left(x_{n+j}, y_{n+j}, y_{n+j}^{\prime}\right)
$$

$$
g_{n+j}=\frac{d f\left(x, y(x), y^{\prime}(x)\right)}{d x},
$$

which by notation is

$$
g_{n+j}=g\left(x_{n+j}, y_{n+j}, y_{n+j}^{\prime}\right) .
$$

Expanding individual terms in (3) using Taylor series expansion and substituting the expansions back in (3) gives the following written in matrix form as below (coefficients of $y^{(m)} x_{n}$ are equated)

$$
\left(\begin{array}{cccccccc}
1 & 1 & 0 & 0 & 0 & 0 & 0 & 0 \\
0 & h & 0 & 0 & 0 & 0 & 0 & 0 \\
0 & \frac{(h)^{2}}{2 !} & 1 & 1 & 1 & 0 & 0 & 0 \\
0 & \frac{(h)^{3}}{3 !} & 0 & h & 2 h & 1 & 1 & 1 \\
0 & \frac{(h)^{4}}{4 !} & 0 & \frac{(h)^{2}}{2 !} & \frac{(2 h)^{2}}{2 !} & 0 & h & 2 h \\
0 & \frac{(h)^{5}}{5 !} & 0 & \frac{(h)^{3}}{3 !} & \frac{(2 h)^{3}}{3 !} & 0 & \frac{(h)^{2}}{2 !} & \frac{(2 h)^{2}}{2 !} \\
0 & \frac{(h)^{6}}{6 !} & 0 & \frac{(h)^{4}}{4 !} & \frac{(2 h)^{4}}{4 !} & 0 & \frac{(h)^{3}}{3 !} & \frac{(2 h)^{3}}{3 !} \\
0 & \frac{(h)^{7}}{7 !} & 0 & \frac{(h)^{5}}{5 !} & \frac{(2 h)^{5}}{5 !} & 0 & \frac{(h)^{4}}{4 !} & \frac{(2 h)^{4}}{4 !}
\end{array}\right)\left(\begin{array}{c}
\alpha_{0} \\
\alpha_{1} \\
\beta_{0} \\
\beta_{1} \\
\beta_{2} \\
\lambda_{0} \\
\lambda_{1} \\
\lambda_{2}
\end{array}\right)=\left(\begin{array}{c}
1 \\
2 h \\
\frac{(2 h)^{2}}{2 !} \\
\frac{(2 h)^{3}}{3 !} \\
\frac{(2 h)^{4}}{4 !} \\
\frac{(2 h)^{5}}{5 !} \\
\frac{(2 h)^{6}}{6 !} \\
\frac{(2 h)^{7}}{7 !}
\end{array}\right) .
$$

The values of $\alpha_{0}, \alpha_{1}, \beta_{0}, \beta_{1}, \beta_{2}, \lambda_{0}, \lambda_{1}$ and $\lambda_{2}$ are obtained using matrix inverse method as given below

$$
\begin{aligned}
& \left(\alpha_{0}, \alpha_{1}, \beta_{0}, \beta_{1}, \beta_{2}, \lambda_{0}, \lambda_{1}, \lambda_{2}\right)^{T} \\
& =\left(-1,2, \frac{2 h^{2}}{15}, \frac{11 h^{2}}{15}, \frac{2 h^{2}}{15}, \frac{h^{3}}{40}, 0,-\frac{h^{3}}{40}\right)^{T} .
\end{aligned}
$$

Substituting (4) in (3) gives the discrete scheme

$$
\begin{aligned}
& y_{n+2}=-y_{n}+2 y_{n+1}+\frac{h^{2}}{15}\left(2 f_{n}+11 f_{n+1}+2 f_{n+2}\right) \\
& +\frac{h^{3}}{40}\left(g_{n}-g_{n+2}\right) .
\end{aligned}
$$

The additional methods needed to obtain the desired block are to be obtained from

$$
\begin{aligned}
& y_{n}^{\prime}=\sum_{j=0}^{1} \alpha_{j} y_{n+j}+\sum_{j=0}^{2} \beta_{j} f_{n+j}+\sum_{j=0}^{2} \lambda_{j} g_{n+j} \\
& y_{n+1}^{\prime}=\sum_{j=0}^{1} \alpha_{j} y_{n+j}+\sum_{j=0}^{2} \beta_{j} f_{n+j}+\sum_{j=0}^{2} \lambda_{j} g_{n+j}, \\
& y_{n+2}^{\prime}=\sum_{j=0}^{1} \alpha_{j} y_{n+j}+\sum_{j=0}^{2} \beta_{j} f_{n+j}+\sum_{j=0}^{2} \lambda_{j} g_{n+j}
\end{aligned}
$$

where each of the terms in (6) are similarly expanded using Taylor series expansion and substituting these expansions back in (6) gives a series of linear equations system that can be solved using matrix inverse approach to obtain the unknown derivatives. 
The substitution of the obtained coefficients into (6) provides the derivatives of the discrete scheme as presented below

$$
\begin{aligned}
& y_{n}^{\prime}=\frac{1}{h}\left(-y_{n}+y_{n+1}\right)+\frac{h}{42}\left(-13 f_{n}-7 f_{n+1}-f_{n+2}\right) \\
& +\frac{h^{2}}{1680}\left(-59 g_{n}+128 g_{n+1}+11 g_{n+2}\right), \\
& y_{n+1}^{\prime}=\frac{1}{h}\left(-y_{n}+y_{n+1}\right)+\frac{h}{1680}\left(187 f_{n}+616 f_{n+1}+37 f_{n+2}\right) \\
& +\frac{h^{2}}{840}\left(16 g_{n}-76 g_{n+1}-5 g_{n+2}\right), \\
& y_{n+2}^{\prime}=\frac{1}{h}\left(-y_{n}+y_{n+1}\right)+\frac{h}{70}\left(11 f_{n}+63 f_{n+1}+31 f_{n+2}\right) \\
& +\frac{h^{2}}{1680}\left(53 g_{n}+128 g_{n+1}-10 g_{n+2}\right) .
\end{aligned}
$$

Combining the equations formulated in the discrete scheme derived in equation (5) together with the derivatives obtained in equation (7), gives an expression that can be presented in the following matrix form

$$
\begin{aligned}
& \left(\begin{array}{cccc}
-2 & 1 & 0 & 0 \\
-\frac{1}{h} & 0 & 0 & 0 \\
-\frac{1}{h} & 0 & 1 & 0 \\
-\frac{1}{h} & 0 & 0 & 1
\end{array}\right)\left(\begin{array}{c}
y_{n+1} \\
y_{n+2} \\
y_{n+1}^{\prime} \\
y_{n+2}^{\prime}
\end{array}\right) \\
& =\left(\begin{array}{c}
-y_{n}+\frac{h^{2}}{15}\left(2 f_{n}+11 f_{n+1}+2 f_{n+2}\right) \\
+\frac{h^{3}}{40}\left(g_{n}-g_{n+2}\right) \\
-y_{n}^{\prime}-\frac{1}{h} y_{n}+\frac{h}{42}\left(-13 f_{n}-7 f_{n+1}-f_{n+2}\right) \\
+\frac{h^{2}}{1680}\left(-58 g_{n}+128 g_{n+1}+11 g_{n+2}\right) \\
-\frac{1}{h} y_{n}+\frac{h}{1680}\left(187 f_{n}+616 f_{n+1}+37 f_{n+2}\right) \\
+\frac{h^{2}}{840}\left(16 g_{n}-76 g_{n+1}-5 g_{n+2}\right) \\
-\frac{1}{h} y_{n}+\frac{h}{70}\left(11 f_{n}+63 f_{n+1}+31 f_{n+2}\right) \\
+\frac{h^{2}}{1680}\left(53 g_{n}+128 g_{n+1}-10 g_{n+2}\right)
\end{array}\right) .
\end{aligned}
$$

Adopting matrix inverse method, $x=A^{-1} B$ where

$$
\begin{gathered}
A=\left(\begin{array}{cccc}
-2 & 1 & 0 & 0 \\
-\frac{1}{h} & 0 & 0 & 0 \\
-\frac{1}{h} & 0 & 1 & 0 \\
-\frac{1}{h} & 0 & 0 & 1
\end{array}\right), \\
B=\left(\begin{array}{c}
-y_{n}+\frac{h^{2}}{15}\left(2 f_{n}+11 f_{n+1}+2 f_{n+2}\right) \\
+\frac{h^{3}}{40}\left(g_{n}-g_{n+2}\right) \\
-y_{n}^{\prime}-\frac{1}{h} y_{n}+\frac{h}{42}\left(-13 f_{n}-7 f_{n+1}-f_{n+2}\right) \\
+\frac{h^{2}}{1680}\left(-58 g_{n}+128 g_{n+1}+11 g_{n+2}\right) \\
-\frac{1}{h} y_{n}+\frac{h}{1680}\left(187 f_{n}+616 f_{n+1}+37 f_{n+2}\right) \\
+\frac{h^{2}}{840}\left(16 g_{n}-76 g_{n+1}-5 g_{n+2}\right) \\
-\frac{1}{h} y_{n}+\frac{h}{70}\left(11 f_{n}+63 f_{n+1}+31 f_{n+2}\right) \\
+\frac{h^{2}}{1680}\left(53 g_{n}+128 g_{n+1}-10 g_{n+2}\right)
\end{array}\right),
\end{gathered}
$$

$x=\left[y_{n+1}, y_{n+2}, y_{n+1}^{\prime}, y_{n+2}^{\prime}\right]^{T}$ is determined and expressed as given below

$$
\begin{aligned}
& y_{n+1}=y_{n}+h y_{n}^{\prime}+\frac{h^{2}}{42}\left(13 f_{n}+7 f_{n+1}+f_{n+2}\right) \\
& +\frac{h^{3}}{1680}\left(59 g_{n}-128 g_{n+1}-11 g_{n+2}\right), \\
& y_{n+2}=y_{n}+2 h y_{n}^{\prime}+\frac{h^{2}}{105}\left(79 f_{n}+112 f_{n+1}+19 f_{n+2}\right) \\
& +\frac{h^{3}}{105}\left(10 g_{n}-16 g_{n+1}-4 g_{n+2}\right), \\
& y_{n+1}^{\prime}=y_{n}^{\prime}+\frac{h}{240}\left(101 f_{n}+128 f_{n+1}+11 f_{n+2}\right) \\
& +\frac{h^{2}}{240}\left(13 g_{n}-40 g_{n+1}-3 g_{n+2}\right), \\
& y_{n+2}^{\prime}=y_{n}^{\prime}+\frac{h}{15}\left(7 f_{n}+16 f_{n+1}+7 f_{n+2}\right)+\frac{h^{2}}{15}\left(g_{n}-g_{n+2}\right) .
\end{aligned}
$$

The block method in equation (8) is the new two-step third-derivative starting formula to implement the Numerov method.

Note that the starting formula proposed by [6] takes the form given below

$$
y_{n+1}=y_{n}+h y_{n}^{\prime}+\frac{h^{2}}{24}\left(7 f_{n}+6 f_{n+1}-f_{n+2}\right) \text {, }
$$

while the starting formula from [1] takes the form

$$
y_{n+1}=y_{n}+h y_{n}^{\prime}+\frac{h^{2}}{360}\left(89 f_{n}+186 f_{n+1}-128 f_{n+\frac{3}{2}}+33 f_{n+2}\right) \text {. }
$$

\section{Basic Properties of the \\ Third-Derivative Block Method}

The properties tested for the block method will be limited to the required to ensure convergence. This follows from the conditions stated by [4] that a linear multistep method is convergent if it is consistent and zero-stable.

Definition 3.1. A linear multistep method is consistent if it has order $p \geq 1$

The obtain the order of the third-derivative block method, the correctors in equation (8) above are considered and the linear operators defined as

$$
\begin{aligned}
& L_{1}=y_{n+1}-\left[\begin{array}{l}
y_{n}+h y_{n}^{\prime}+\frac{h^{2}}{42}\left(13 f_{n}+7 f_{n+1}+f_{n+2}\right) \\
+\frac{h^{3}}{1680}\left(59 g_{n}-128 g_{n+1}-11 g_{n+2}\right)
\end{array}\right], \\
& L_{2}=y_{n+2}-\left[\begin{array}{l}
y_{n}+2 h y_{n}^{\prime}+\frac{h^{2}}{105}\left(79 f_{n}+112 f_{n+1}+19 f_{n+2}\right) \\
+\frac{h^{3}}{105}\left(10 g_{n}-16 g_{n+1}-4 g_{n+2}\right)
\end{array}\right]
\end{aligned}
$$

following the concept of [8].

Expanding individual terms in (11) using Taylor series expansions defined as 


$$
\begin{aligned}
y_{n+j}= & y\left(x_{n}+j h\right)=y\left(x_{n}\right)+(j h) y^{\prime}\left(x_{n}\right) \\
& +\frac{(j h)^{2}}{2 !} y^{\prime \prime}\left(x_{n}\right)+\ldots \\
f_{n+j}= & y^{\prime \prime}\left(x_{n}+j h\right)=y^{\prime \prime}\left(x_{n}\right)+(j h) y^{\prime \prime \prime}\left(x_{n}\right)+\ldots \\
g_{n+j}= & y^{\prime \prime \prime}\left(x_{n}+j h\right)=y^{\prime \prime \prime}\left(x_{n}\right)+(j h) y^{i v}\left(x_{n}\right)+\ldots
\end{aligned}
$$

Then substituting (12) in (11) and collecting like terms gives (11) in the form

$$
\begin{aligned}
& L_{1}=C_{0} y(x)+C_{1} h y^{\prime}\left(x_{n}\right)+\ldots+C_{p} h^{p} y^{(p)}\left(x_{n}\right)+\ldots \\
& L_{2}=C_{0} y(x)+C_{1} h y^{\prime}\left(x_{n}\right)+\ldots+C_{p} h^{p} y^{(p)}\left(x_{n}\right)+\ldots
\end{aligned}
$$

To satisfy Definition 3.1, the block method has order $p \geq 1$ if $C_{3}=0$ since the error constant for the block method is expected at $C_{p+2} \neq 0$.

Thus for the expression of $L_{1}$,

$$
\begin{aligned}
& C_{0}=1-1=0 \\
& C_{1}=1-1=0 \\
& C_{2}=\frac{1}{2 !}-\frac{13}{42}-\frac{7}{42}-\frac{1}{42}=0 \\
& C_{3}=\frac{1}{3 !}-\frac{7}{42}-\frac{2}{42}-\frac{59}{1680}+\frac{128}{1680}+\frac{11}{1680}=0
\end{aligned}
$$

and for expression $L_{2}$

$$
\begin{aligned}
& C_{0}=1-1=0 \\
& C_{1}=2-2=0 \\
& C_{2}=\frac{2^{2}}{2 !}-\frac{79}{105}-\frac{112}{105}-\frac{19}{105}=0 \\
& C_{3}=\frac{2^{3}}{3 !}-\frac{112}{105}-\frac{38}{105}-\frac{10}{105}+\frac{16}{105}+\frac{4}{105}=0
\end{aligned}
$$

Hence, the block method is consistent.

Moving on to the property of zero-stability, first equation (11) is coined to take the form

$$
I_{2} Y_{n k}=A_{1} Z_{n}+B_{1} F_{n}+B_{2} F_{n k}+C_{1} G_{n}+C_{2} G_{n k}
$$

where

$$
\begin{aligned}
& I_{2}=\left(\begin{array}{cc}
1 & 0 \\
0 & 1
\end{array}\right), Y_{n k}=\left(\begin{array}{c}
y_{n+1} \\
y_{n+2}
\end{array}\right), A_{1}=\left(\begin{array}{ll}
0 & 1 \\
0 & 1
\end{array}\right), \\
& Z_{n}=\left(\begin{array}{c}
y_{n-1}^{\prime} \\
y_{n}^{\prime}
\end{array}\right), B_{1}=\left(\begin{array}{ll}
0 & \frac{13}{42} \\
0 & \frac{79}{105}
\end{array}\right), F_{n}=\left(\begin{array}{c}
f_{n-1} \\
f_{n}
\end{array}\right), \\
& B_{2}=\left(\begin{array}{cc}
\frac{7}{42} & \frac{1}{42} \\
\frac{112}{105} & \frac{19}{105}
\end{array}\right), F_{n k}=\left(\begin{array}{c}
f_{n+1} \\
f_{n+2}
\end{array}\right), C_{1}=\left(\begin{array}{cc}
0 & \frac{59}{1680} \\
0 & \frac{10}{105}
\end{array}\right), \\
& G_{n}=\left(\begin{array}{c}
g_{n-1} \\
g_{n}
\end{array}\right), C_{2}=\left(\begin{array}{cc}
-\frac{128}{1680} & -\frac{11}{1680} \\
-\frac{16}{105} & -\frac{4}{105}
\end{array}\right), G_{n k}=\left(\begin{array}{c}
g_{n+1} \\
g_{n+2}
\end{array}\right)
\end{aligned}
$$

Now, the third-derivative block method is zero-stable if the roots of the characteristic polynomial $p(r)=\left|r I_{2}-A_{1}\right|$ are simple or less than one. Upon substitution,

$$
p(r)=\left|r\left(\begin{array}{ll}
1 & 0 \\
0 & 1
\end{array}\right)-\left(\begin{array}{ll}
0 & 1 \\
0 & 1
\end{array}\right)\right|=r(r-1)
$$

with roots $r=0,1$ and this implies the block method is zerostable.

On satisfying all criteria for convergence, the third-derivative block method is said to be convergent and hence suitable for application to solve ordinary differential equations.

\section{Numerical Results}

In this section, two second order ordinary differential equations will be considered, which are already solved in literature by past authors. Comparison will be made by computing the absolute value difference between the exact solution and the computed solution.

Problem 1

$$
y^{\prime \prime}=-y, y(0)=1=y^{\prime}(0), h=0.1
$$

Exact Solution: $y(x)=\cos x+x \sin x$

\section{Source:[1]}

\section{Problem 2}

$$
y^{\prime \prime}=100 y, y(0)=1, y^{\prime}(0)=-10, h=0.01
$$

Exact Solution: $y(x)=e^{-10 x}$

Source: [1]

Table 1. Absolute Errors for Problem 1 where starting values are provided for the Numerov method using (8), (9) and (10).

\begin{tabular}{llll}
\hline$x$ & Starting Value (9) & Starting Value (10) & Starting Value (8) \\
\hline 0.10 & $2.07 \mathrm{E}-07$ & $6.92 \mathrm{E}-09$ & $6.28 \mathrm{E}-13$ \\
0.20 & $4.07 \mathrm{E}-07$ & $1.76 \mathrm{E}-08$ & $4.56 \mathrm{E}-09$ \\
0.30 & $6.12 \mathrm{E}-07$ & $1.62 \mathrm{E}-08$ & $4.54 \mathrm{E}-09$ \\
0.40 & $8.17 \mathrm{E}-07$ & $4.37 \mathrm{E}-08$ & $9.69 \mathrm{E}-09$ \\
0.50 & $1.02 \mathrm{E}-06$ & $1.20 \mathrm{E}-07$ & $9.55 \mathrm{E}-09$ \\
0.60 & $1.22 \mathrm{E}-06$ & $1.87 \mathrm{E}-07$ & $1.50 \mathrm{E}-08$ \\
0.70 & $1.41 \mathrm{E}-06$ & $3.07 \mathrm{E}-07$ & $1.46 \mathrm{E}-08$ \\
0.80 & $1.60 \mathrm{E}-06$ & $4.19 \mathrm{E}-07$ & $2.00 \mathrm{E}-08$ \\
0.90 & $1.76 \mathrm{E}-06$ & $5.79 \mathrm{E}-07$ & $1.93 \mathrm{E}-08$ \\
1.00 & $1.92 \mathrm{E}-06$ & $7.27 \mathrm{E}-07$ & $2.43 \mathrm{E}-08$ \\
\hline
\end{tabular}

Table 2. Absolute Errors for Problem 2 where starting values are provided for the Numerov method using (8), (9) and (10).

\begin{tabular}{llll}
\hline$x$ & Starting Value (9) & Starting Value (10) & Starting Value (8) \\
\hline 0.10 & $2.04 \mathrm{E}-07$ & $4.96 \mathrm{E}-09$ & $5.29 \mathrm{E}-13$ \\
0.20 & $4.15 \mathrm{E}-07$ & $1.49 \mathrm{E}-08$ & $3.77 \mathrm{E}-09$ \\
0.30 & $6.33 \mathrm{E}-07$ & $6.68 \mathrm{E}-09$ & $3.78 \mathrm{E}-09$ \\
0.40 & $8.61 \mathrm{E}-07$ & $2.40 \mathrm{E}-08$ & $6.93 \mathrm{E}-09$ \\
0.50 & $1.10 \mathrm{E}-06$ & $6.77 \mathrm{E}-07$ & $7.04 \mathrm{E}-09$ \\
0.60 & $1.35 \mathrm{E}-06$ & $1.08 \mathrm{E}-07$ & $9.74 \mathrm{E}-09$ \\
0.70 & $1.62 \mathrm{E}-06$ & $1.77 \mathrm{E}-07$ & $1.00 \mathrm{E}-08$ \\
0.80 & $1.91 \mathrm{E}-06$ & $2.32 \mathrm{E}-07$ & $1.24 \mathrm{E}-08$ \\
0.90 & $2.22 \mathrm{E}-06$ & $3.13 \mathrm{E}-07$ & $1.29 \mathrm{E}-08$ \\
1.00 & $2.56 \mathrm{E}-06$ & $3.95 \mathrm{E}-07$ & $1.52 \mathrm{E}-08$ \\
\hline
\end{tabular}




\section{Discussion of Results}

These numerical problems considered are the same considered in the work of [1]. With reference to Table 1 and Table 2 above, although the starting value adopted by [1] as seen in equation (10) is also a two-step method but with one hybrid point $\frac{3}{2}$, this two-step third-derivative method performs better having closer accuracy to the exact solution. In the same vein, the two-step third-derivative method also gives more accurate results than the two step starting formula by [6] as stated in equation (9).

\section{Conclusion}

This paper presents a new approach for starting Numerov method with better accuracy. The starting method presented by $[1,6]$ and the third-derivative method presented in this paper are all two-step methods which gives a good basis for the comparison of these starting values. However, from the results in Tables 1 and 2, the accuracy of the new starting formula on the Numerov block method shows better accuracy. Hence, this new starting method (third-derivative block method) is a better formula for adequately starting the Numerov method.

\section{References}

[1] Adee, S. O., P. Onumanyi, U. W. Sirisena and Y. A. Yahaya. 2005. Note on starting the Numerov method more accurately by a hybrid formula of order four for an initial value problem. Journal of Computational and Applied Mathematics, 175 (2): 369-373. doi: 10.1016/j.cam.2004.06.016.

[2] Ehle, B. L. 1968. High order A-stable methods for the numerical solution of systems of DE's. BIT Numerical Mathematics, 8 (4), 276-278. doi: 10.1007/BF01933437.

[3] Enright, W. H. 1974. Second derivative multistep methods for stiff ordinary differential equations. SIAM Journal on Numerical Analysis, 11 (2), 321-331. doi: 10.1137/0711029.

[4] Fatunla, S. O. 1988. Numerical methods for initial value problems in ordinary differential equations, Academic Press, New York.

[5] Jator, S. N., and Li, J.. 2012. An algorithm for second order initial and boundary value problems with an automatic error estimate based on a third derivative method. Numerical Algorithms, 59 (3), 333-346., doi: 10.1007/s11075-011-9492-3.
[6] Gonzalez, J. Q. and D. Thompson. 1997. Getting started with Numerovs method. Computers in Physics, 11 (5): 514-515. http://dx.doi.org/10.1063/1.168593.

[7] Konguetsof, A. 2010. A new two-step hybrid method for the numerical solution of the Schrodinger equation. Journal of Mathematical Chemistry, 47 (2): $871-890$. doi: 10.1007/s10910-009-9606-5.

[8] Lambert, J. D. Computational methods in ordinary differential equations, Wiley: London, 1973.

[9] Mishra, B. N. and R. K. Mohanty. 2013. Single cell Numerov type discretization for $2 \mathrm{D}$ biharmonic and triharmonic equations on unequal mesh. Journal of Mathematical and $\begin{array}{llll}\text { Computational Science, } & 3 & \text { (1): } 242-253 .\end{array}$ http://www.scik.org/index.php/jmcs/article/viewFile/774/308.

[10] Mohanty, R. K. and R. Kumar. 2014. A novel numerical algorithm of Numerov Type for 2D quasi-linear elliptic boundary value problems. International Journal for Computational Methods in Engineering Science and $\begin{array}{llll}\text { Mechanics, } & 15 & \text { (6): } & 473-489 .\end{array}$ 10.1080/15502287.2014.934488.

[11] Norton, M. S. 2009. Numerov's Method for approximating solutions to Poisson's equation. https://www.siue.edu/_mnorton/Numerov.pdf (Accessed on October 29, 2015).

[12] Obrechkoff, N. 1942. On mechanical quadrature (Bulgarian French summary). Spisanie Bulgar. Akad. Nauk, 65, 191-289.

[13] Onumanyi, P., U. W. Sirisena and S. Adee. 2002. Some theoretical considerations of continuous linear multistep methods for $u^{(v)}=f(x, u), v=1,2$. Bagale Journal of Pure and Applied Sciences, 2 (2): 1-5.

[14] Sahi, R. K., Jator, S. N., \& Khan, N. A. 2013. Continuous fourth derivative method for third order boundary value problems. International journal of pure and applied mathematics, 85 (5), 907-923. doi: 10.12732/ijpam.v85i5.9.

[15] Simos, T. E. 2009. A new Numerov-type method for the numerical solution of the Schrodinger equation. Journal of Mathematical Chemistry, 46 (3): 981-1007. doi: 10.1007/s10910-009-9553-1.

[16] Yusuph, Y. and P. Onumanyi. 2005. New multiple FDMs through multistep collocation for $y=f(x, y)$. Proceedings of the National Mathematical Center, Abuja Nigeria.

[17] Vigo-Aguiar, J. and H. Ramos. 2005. A variable-step Numerov method for the numerical solution of the Schrodinger equation. Journal of Mathematical Chemistry, 37 (3): 255-262. doi: 10.1007/s10910-004-1467-3. 